\title{
Warm-white light-emitting diodes integrated with colloidal quantum dots for high luminous efficacy and color rendering
}

Article in Optics Letters · October 2010

DOI: 10.1364/OL.35.003372 · Source: PubMed

CITATIONS

52

4 authors, including:

\section{Sedat Nizamoglu}

Harvard University

75 PUBLICATIONS 1,449 CITATIONS

SEE PROFILE

\section{X.W. Sun}

Nanyang Technological University

642 PUBLICATIONS 16,182 CITATIONS

SEE PROFILE
READS

77

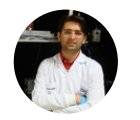

Talha Erdem

University of Cambridge

43 PUBLICATIONS 585 CITATIONS

SEE PROFILE

Some of the authors of this publication are also working on these related projects: 


\title{
Warm-white light-emitting diodes integrated with colloidal quantum dots for high luminous efficacy and color rendering
}

\author{
Sedat Nizamoglu, ${ }^{1}$ Talha Erdem, ${ }^{1}$ Xiao Wei Sun, ${ }^{2}$ and Hilmi Volkan Demir ${ }^{1,2, *}$ \\ ${ }^{1}$ Department of Electrical and Electronics Engineering, Department of Physics, and UNAM-Institute \\ of Materials Science and Nanotechnology, Bilkent University, Ankara, Turkey 06800 \\ ${ }^{2}$ School of Electrical and Electronic Engineering, Microelectronics Division; School of Physical and Mathematical Sciences, \\ Physics and Applied Physics Division, Nanyang Technological University, Singapore 639798 \\ *Corresponding author: volkan@stanfordalumni.org
}

Received June 2, 2010; revised September 1, 2010; accepted September 2, 2010; posted September 14, 2010 (Doc. ID 129472); published October 8, 2010

Warm-white LEDs (WLEDs) with high spectral quality and efficiency are required for lighting applications, but current experimental performances are limited. We report on nanocrystal quantum dot (NQD) hybridized WLEDs with high performance that exhibit a high luminous efficacy of optical radiation exceeding $350 \mathrm{~lm} / \mathrm{W}_{\text {opt }}$ and a high color rendering index close to 90 at a low correlated color temperature $<3000 \mathrm{~K}$. These spectrally engineered WLEDs are obtained using a combination of CdSe/ZnS core/shell NQD nanophosphors integrated on blue InGaN/GaN LEDs. (c) 2010 Optical Society of America

OCIS codes: $160.4236,230.3670,250.5230$.

Theoretical emission spectra of white LEDs (WLEDs) have been investigated to achieve efficient solid-state lighting (SSL) with a high color rendering index (CRI) approaching 90 and a luminous efficacy of optical radiation (LER) higher than $350 \mathrm{~lm} / \mathrm{W}$ [1-4]. However, the reported sets of experimental LER of optical radiation and CRI have been limited [ [5-7]. A high CRI approaching 90 is important for general purpose lighting. A high LER exceeding $350 \mathrm{~lm} / \mathrm{W}$ is also essential to ensure that the light source provides an efficient spectral content for the human eye. As the current state of the art, a warm-WLED with an LER of $274 \mathrm{~lm} / \mathrm{W}_{\text {opt }}$ and a CRI of 89 at a correlated color temperature (CCT) of $3100 \mathrm{~K}$ has previously been demonstrated by using nitride-based $\mathrm{Eu}^{2+}$ phosphors on a blue LED [5]. For the current study, to outperform conventional phosphors in terms of LER, we developed photometric models, made new WLED designs, and experimentally demonstrated warm-WLEDs combined with nanophosphors of semiconductor nanocrystal quantum dots (NQDs) on LED chips to achieve LER $>350 \mathrm{~lm} / \mathrm{W}_{\text {opt }}$ with CRI $=89.2$ at CCT $<3000 \mathrm{~K}$.

In lighting applications, NQDs offer important benefits for color conversion LEDs. Their emission properties can be conveniently adjusted through the quantum size effect and by using different material systems and shapes [7]. Furthermore, NQDs can be easily integrated on substrates using common techniques (e.g., spin coating and layer-by-layer assembly), and they favorably exhibit high quantum efficiency, photostability, and photobleaching thresholds [8]. Furthermore, they provide high spectral purity because of their narrow emission linewidths. These favorable properties enable us to custom design a desired emission spectrum by using proper combinations of differently sized NQDs [9,10]. Because of these important benefits and continuing developments in their colloidal synthesis, NQDs are strong candidates for use in color conversion LEDs, the operation of which is based on the collective emissions of both LED electroluminescence and NQD photoluminescence [11-13].
Since we use nanocrystals in a host medium [poly (methyl methacrylate)], the environmental and dipoledipole interactions prevent us from exactly predicting the peak emission wavelength shifts, emission broadenings, and relative emission strengths. Thus, we need to perform a numerical analysis to study whether the intended high-quality white-light generation can be achieved by using our green-, yellow-, and red-emitting CdSe/ZnS core/shell NQDs. These nanocrystals are coated with long chain amine capping agents and exhibit respective photoluminescence peak wavelengths at 528 , 560 , and $609 \mathrm{~nm}$ in toluene. We employed the emission spectrum of each color source as a Gaussian function [14] and changed the emission peaks of each color with a $10 \mathrm{~nm}$ step size in the range of $450-470 \mathrm{~nm}$ for blue, $535-555 \mathrm{~nm}$ for green, $557-577 \mathrm{~nm}$ for yellow, and 610-630 nm for red. Furthermore, we varied the FWHM of the blue emission with a $10 \mathrm{~nm}$ step size from 25 to $55 \mathrm{~nm}$ and used a FWHM variation from 30 to $50 \mathrm{~nm}$ with the other color components. We also varied the peak amplitudes of each color from 430 to 470 units for blue, 750 to 790 units for green, and 470 to 510 units for yellow, with a 20 unit step size; for red, the variation was set between 1400 and 1500 units with a 50 unit step size. As a result, we generated and simulated a total number of $1,180,980$ hybrid LED designs using experimentally realistic input parameters in our simulations. In this analysis, we optimized our WLED performances for daytime vision (with a peak eye sensitivity response at $555 \mathrm{~nm}$ in the photopic mode) rather than for nighttime vision (with a peak eye sensitivity response at $507 \mathrm{~nm}$ in scotopic mode due to the Purkinje shift). To understand feasible performance of LEDs using these specific NQDs, the simulation results of CRI versus LER are presented in Fig. 1(a), including only those with the resulting photometric performances of LER $>300 \mathrm{~lm} / \mathrm{W}_{\text {opt }}$ and CRI > 60. Consequently, these simulation results show that our NQD nanophosphors are capable of achieving spectrally efficient and high-quality white-light generation. 


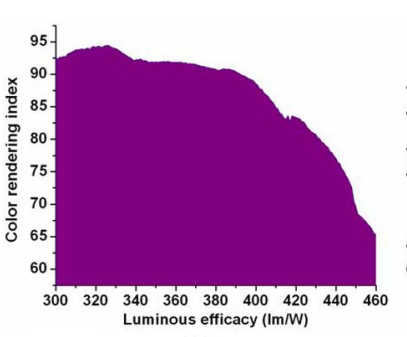

(a)

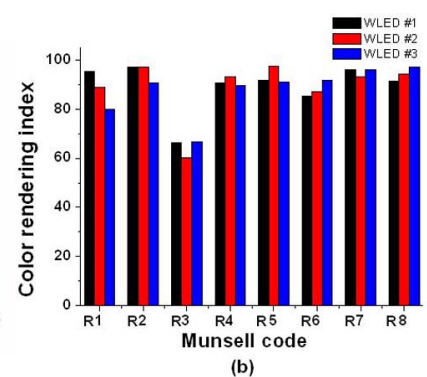

(b)
Fig. 1. (Color online) (a) Feasible sets of CRI versus LER for WLEDs integrated with NQD nanophosphors and (b) CRI of NQD-integrated warm-WLEDs (WLEDs 1, 2, and 3) analyzed at different Munsell hues.

For our first design of NQD-integrated WLEDs (WLED 1), we hybridized $31.91 \mathrm{nmol}$ of green-emitting NQDs, $1.42 \mathrm{nmol}$ of yellow-emitting NQDs, and $0.37 \mathrm{nmol}$ of orange-emitting NQDs on our blue LED. The orangeemitting nanocrystals decrease the color temperature for warm-white-light generation and balance the luminescence of the green-emitting nanocrystals along with the blue LED. As a result, the chromaticity coordinates remain inside the white region while keeping LER and CRI high. Figure 2 presents the resulting collective emission and chromaticity properties of the hybrid LED at different current injection levels. The operating point at $12 \mathrm{~mA}$ corresponds to $(x, y)=(0.425,0.378), \mathrm{LER}=$ $357 \mathrm{~lm} / \mathrm{W}_{\mathrm{opt}}, \mathrm{CRI}=89.2$, and CCT $=2982 \mathrm{~K}$. This makes a warm-WLED with a desirably low CCT of less than $3000 \mathrm{~K}$. This NQD-LED also satisfies the CRI need for future SSL applications (with a CRI > 80) [6]. Furthermore, this hybrid LED reaches an LER of $357 \mathrm{~lm} / \mathrm{W}_{\text {opt }}$, achieving a high spectral efficiency, because these nanocrystal emitters enable us to reduce the deep-red emission at wavelengths longer than $650 \mathrm{~nm}$, where the eye sensitivity function quickly decays and the luminous efficacy decreases in the case of broad emitters with emission tails toward long wavelengths. To investigate the device power conversion efficiency, we use the following equation [14]:

$$
\eta_{\text {power }}=t \cdot \eta_{\mathrm{LED}}+(1-t) \cdot \eta_{\mathrm{NQD}} \cdot\left(\lambda_{\mathrm{LED}} / \lambda_{\mathrm{NQD}}\right),
$$

where $\eta_{\text {power }}$ is the power conversion efficiency of the NQD-integrated LED, $t$ is the power fraction of the transmitted radiation of the blue LED, $\eta_{\mathrm{LED}}$ is the external quantum efficiency of the blue LED, $\eta_{\mathrm{NQD}}$ is the quantum efficiency of the nanocrystal quantum dots, and $\lambda_{\mathrm{LED}}$ and $\lambda_{\mathrm{NQD}}$ are the center emission wavelengths of blue LED and the nanocrystal quantum dots, respectively. For our calculation, we used the following values for our device parameters: $\lambda_{\mathrm{LED}}=452 \mathrm{~nm}, \lambda_{\mathrm{NQD}}=588.4 \mathrm{~nm}, \eta_{\mathrm{LED}}=$ $0.10, \eta_{\mathrm{NQD}}=0.15$, and $t=0.20$, which results in a power conversion efficiency of 0.11 . Thus, this hybrid WLED achieves a luminous efficiency of approximately $40 \mathrm{~lm} /$ $\mathrm{W}_{\text {electrical }}$. Also, we predict that a luminous efficiency of $100 \mathrm{~lm} / \mathrm{W}_{\text {electrical }}$ is attainable by using optimized blue LEDs and nanocrystals, as it is possible to obtain $\eta_{\mathrm{LED}}=$ 0.50 and $\eta_{\mathrm{NQD}}=0.35$.

For our second set of NQD-based WLEDs (WLED 2), we integrated $31.91 \mathrm{nmol}$ of green-emitting, $1.42 \mathrm{nmol}$ of yellow-emitting, and $0.55 \mathrm{nmol}$ of orange-emitting NQDs.

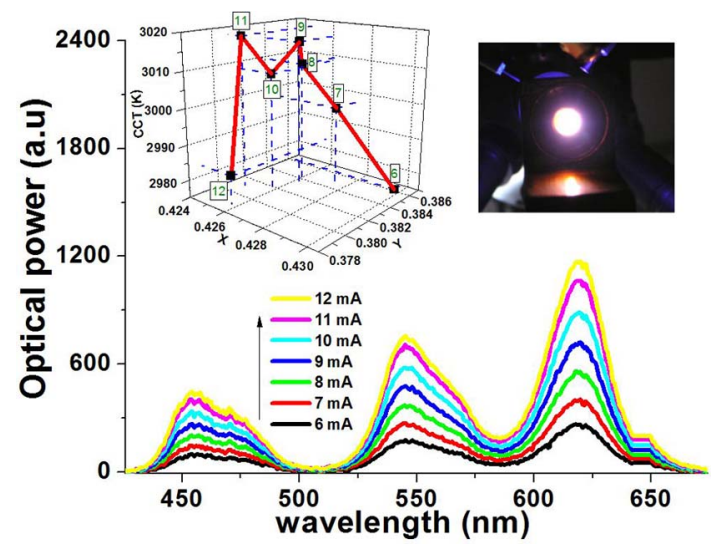

Fig. 2. (Color online) Electroluminescence spectra of the first NQD-LED design (WLED 1) integrated with green-, yellow-, and orange-emitting CdSe/ZnS core/shell NQD nanophosphors on blue LED chips $\left(\lambda_{\mathrm{EL}}=452 \mathrm{~nm}\right)$ driven at different levels of current injection at room temperature, along with the corresponding $(x, y)$ coordinates versus CCT and a picture of a NQD-LED generating white light. In the inset, the values given in the squares represent the current injection levels at that operating point.

We measured the emission spectra of this hybrid LED with the corresponding optical properties at different injected current levels, as shown in Fig. 3. The experimental operating point at $12 \mathrm{~mA}$ led to $(x, y)=(0.445,0.382)$, with $\quad \mathrm{LER}=349 \mathrm{~lm} / \mathrm{W}_{\text {opt }}, \quad \mathrm{CRI}=88.9, \quad$ and $\quad \mathrm{CCT}=$ $2781 \mathrm{~K}$. This second set of results corresponds to a warm-WLED with a lower CCT, because the optical intensity coming from orange-emitting nanocrystals is increased. The CRI, despite a slight decrease, is 88.9, satisfying the need for CRI $>80$ for future WLEDs. The LER is $349 \mathrm{~lm} / \mathrm{W}_{\text {opt }}$, which is also higher than the previous best results of conventional phosphors [5].

In our last set of NQD-integrated WLEDs (WLĒD 3), we incorporated $31.91 \mathrm{nmol}$ of green-emitting, $1.42 \mathrm{nmol}$ of yellow-emitting, and $0.74 \mathrm{nmol}$ of orange-emitting NQDs. We obtained the collective emission spectra and optical properties at various current injection levels, as depicted

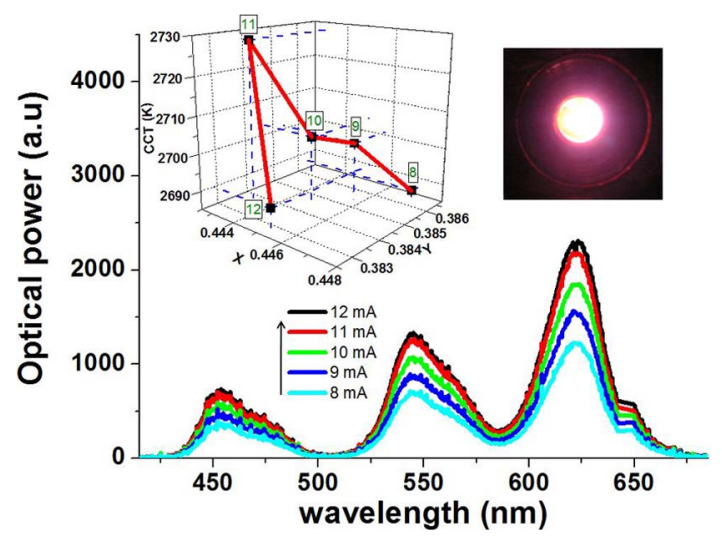

Fig. 3. (Color online) Electroluminescence spectra of the second NQD-LED design (WLED 2) integrated with green-, yellow-, and orange-emitting CdSe/ZnS core/shell NQD nanophosphors on blue LED chips $\left(\lambda_{\mathrm{EL}}=452 \mathrm{~nm}\right)$ driven at different levels of current injection at room temperature, along with the corresponding $(x, y)$ coordinates versus CCT and a picture of a NQD-LED generating white light. In the inset, the values given in the squares represent the current injection levels at that operating point. 


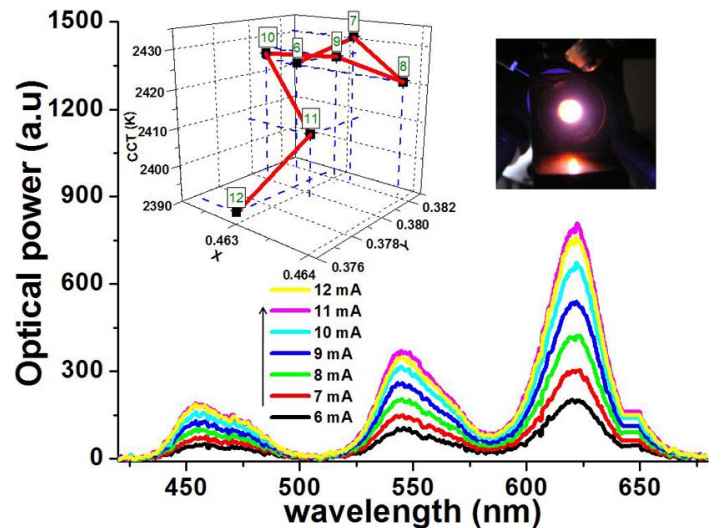

Fig. 4. (Color online) Electroluminescence spectra of the third NQD-LED design (WLED 3) integrated with green-, yellow-, and orange-emitting CdSe/ZnS core/shell NQD nanophosphors on blue LED chips $\left(\lambda_{\mathrm{EL}}=452 \mathrm{~nm}\right)$ driven at different levels of current injection at room temperature, along with the corresponding $(x, y)$ coordinates versus CCT and a picture of NQD-LED while generating white light. In the inset, the values given in the squares represent the current injection levels at that operating point.

in Fig. 4. The emission spectrum at $12 \mathrm{~mA}$ led to

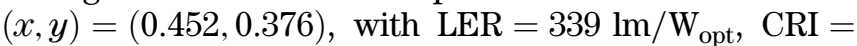
87.8 , and CCT $=2390 \mathrm{~K}$. For this sample we achieved a warm-WLED with an even lower CCT of $2390 \mathrm{~K}$. The CRI and luminous efficacy slightly decreased to 87.8 and $339 \mathrm{~lm} / \mathrm{W}_{\text {opt }}$, respectively. Nevertheless, this warmWLED still meets high LER and CRI requirements.

In all of these three implementations (WLEDs 1-3), it is worth mentioning that we observe a slight shift of both chromaticity coordinates and CCT in response to a changing current injection level. However, these small changes are difficult to clearly distinguish by the naked human eye. These WLEDs exhibit reasonable color stability under the reported current injection levels. We further analyzed the experimental CRI performance of WLEDs 1-3 at each Munsell color, as shown in Fig. 1(b). These internationally accepted Munsell test color samples are defined according to their spectral reflectivity [14]. The CRI is calculated by taking the average color rendering of each Munsell code. The CRI for each Munsell code is above 80, except for the Munsell color of R3. At R3, there is a decrease corresponding to halfway between the green and yellow color spectral ranges. This means that there is room for improvement in CRI by increasing the color rendering capability of the greenyellow color range.
To summarize, we developed nanocrystal integrated warm-WLEDs with high luminous efficacy of optical radiation and high CRI at the same time. These NQDhybridized WLEDs demonstrated significantly improved spectral performance with LER of $357 \mathrm{~lm} / \mathrm{W}_{\text {opt }}$ and CRI of 89.2 at CCT of $2982 \mathrm{~K}$. These hybrid LEDs hold great promise for future SSL applications.

We acknowledgefinancial support from the National Research Foundation (NRF-RF-2009-09), European Science Foundation-European Young Investigator Awards, European Commission Framework Program (EC FP7 N4E-NoE), Turkish Academy of Sciences-Young Scientists Award Programme, Scientific and Technological Research Council of Turkey (TUBITAK)-Department of Scientist Support, and TUBITAK (107E088, 109E002, 109E004, and 110E010).

\section{References}

1. J. M. Phillips, M. E. Coltrin, M. H. Crawford, A. J. Fischer, M. R. Krames, R. Mueller-Mach, G. O. Mueller, Y. Ohno, L. E. S. Rohwer, J. A. Simmons, and J. Y. Tsao, Laser Photon. Rev. 1, 307 (2007).

2. J. Y. Tsao, in The 21st Annual Meeting of the IEEE Lasers and Electro-Optics Society Conference Proceedings (IEEE, 2008), paper WU4.

3. A. Žukauskas, R. Vaicekauskas, F. Ivanauskas, R. Gaska, and M. S. Shur, Appl. Phys. Lett. 80, 234 (2002).

4. T. Erdem, S. Nizamoglu, X. W. Sun, and H. V. Demir, Opt. Express 18, 340 (2010).

5. M. R. Krames, O. B. Shchekin, R. Mueller-Mach, G. O. Mueller, L. Zhou, G. Harbers, and M. G. Craford, J. Display Technol. 3, 160 (2007).

6. S. Nizamoglu, G. Zengin, and H. V. Demir, Appl. Phys. Lett. 92, 031102 (2008).

7. V. L. Colvin, M. C. Schlamp, and A. P. Alivisatos, Nature 370, 354 (1994).

8. R. Osovsky, D. Cheskis, V. Kloper, A. Sashchiuk, M. Kroner, and E. Lifshitz, Phys. Rev. Lett. 102, 197401 (2009).

9. C. B. Murray, C. R. Kagan, and M. G. Bawendi, Annu. Rev. Mater. Sci. 30, 545 (2000).

10. S. V. Gaponenko, Introduction to Nanophotonics (Cambridge U. Press, 2010).

11. S. Nizamoglu, T. Ozel, E. Sari, and H. V. Demir, Nanotechnology 18, 065709 (2007).

12. H. Chen, D. Yeh, C. Lu, C. Huang, W. Shiao, J. Huang, C. C. Yang, I. Liu, and W. Su, IEEE Photonics Technol. Lett. 18, 1430 (2006).

13. H. S. Jang, H. Yang, S. W. Kim, J. Y. Han, S. G. Lee, and D. Y. Jeon, Adv. Mater. 20, 2696 (2008).

14. E. F. Schubert, Light Emitting Diode (Cambridge U. Press, 2006). 